\title{
Die Bestimmung der tellurigen Säure bei Gegenwart von Haloïdsalzen.
}

\author{
Von \\ F. A. Gooch und C. A. Pererrs. ${ }^{1}$
}

Tellurige Säure lälst sich, wie BRAUNER ${ }^{2}$ nachgewiesen hat, so bestimmen, dafs man sie zunächst mit einem Überschufs von Kaliumpermanganat (in saurer oder alkalischer Lösung) oxydiert, sodann die höheren Manganoxyde oder den Permanganatüberschuls mit Oxalsäure von bestimmtem Gehalt in schwefelsaurer Lösung zerstört und schliefslich die überschüssige Oxalsäure mit Permanganat zurücktitriert. Da das Permanganat jedoch geneigt ist, in stark schwefelsaurer Lösung (die man notwendigerweise verwenden mufs, um die tellurige Säure dauernd in Lösung zu haiten) zu viel Sauerstoff abzugeben, so mufs bei dieser Methode eine beträchtliche Korrektion ${ }^{3}$ angebracht werden. Wenn man dagegen das Tellurdioxyd ursprünglich in einem Alkalihydroxyd löst und die Lösung entweder vor oder nach der Oxydation durch das Permanganat bis zu einem bestimmten Grade ansäuert, so ist es nicht notwendig, eine Korrektion anzuwenden, wie in einer früheren Mitteilung aus diesem Laboratorium $^{4}$ nachgewiesen wurde. Wenn man z. B. zur alkalischen Lösung überschüssiges Permanganat hinzufügt, sodann die überschüssige Oxalsäure und nicht mehr als $5 \mathrm{cem}$ einer verdünnten Schwefelsüure $\left[1 \mathrm{H}_{2} \mathrm{SO}_{4}: 1 \mathrm{H}_{2} \mathrm{O}\right]$ zugiebt und nun (nach dem Erhitzen auf $80^{\circ} \mathrm{C}$.) die Oxalsäure mit Permanganat zurücktitriert, so erhält man Resultate, die nicht auf eine zu weitgehende Zersetzung des Permanganats schliefsen lassen. Arbeitet man nun in derselben Weise,

${ }^{1}$ Ins Deutsche übertragen von J. Koppes.

2 Journ. Chem. Soc. 1891, 238.

${ }^{8}$ 1. e. 249.

${ }^{4}$ Gooch und Danner, Amer. Journ. of Art. u. Sc. [Sill.] 44, 301. 
wie soeben angegeben, nur mit der Abänderung, dals man vor dem Zusatz des Permanganats die alkalische Lösung mit Schwefelsäure (1:1) ansäuert, jedoch nach dem Wiederauflösen des entstehenden Niederschlages nur noch $1 \mathrm{ccm}$ derselben zusetzt, so erhält man theoretisch richtige Resultate, die auch mit den Werten, welche nach der weiter oben beschriebenen Methode erhalten wurden, übereinstimmen.

In Gegenwart freier Salzsäure verläuft nach den Angaben von Brauner $^{1}$ die Einwirkung des Permanganats auf die tellurige Säure vollkommen unregelmäfsig; dieser unregelmäfsige Reaktionsverlauf kann jedoch nicht (wie bei der Titration von Ferrosalzen in Gegenwart von Salzsäure) durch den Zusatz von Mangansalzen nach dem bekannten Verfahren von KessueR ${ }^{2}$ und ZImmiriranN ${ }^{3}$ korrigiert werden.

Dạgegen scheint eine genaue Bestimmung der tellurigen Säure auch in Gegenwart von Chloriden ohne weiteres möglich zu sein, wenn man die erste Oxydation mit Permanganat in alkalischer Lösung vornimmt und bei der zweiten Titration genau alle Versuchsbedingungen einhält, die für eine korrekte Titration der Oxalsäure mit Permanganat in Gegenwart von Salzsäure notwendig sind; denn die Gefahr einer zu weit gehenden Reduktion des Permanganats kann nicht auftreten, weil die Lösung alkalisch ist, andererseits existiert auch diese Fehlerquelle nicht, wenn alles Tellurit in Tellurat verwandelt ist und vor dem Ansäuern der Lösung. Bezüglich der genauen Bedingungen für die Titration von Oxalsäure mit Permanganat haben wir kürzlich mitgeteilt," dafs bei Gegenwart einer beträchtlichen Salzsäuremenge in der Lösung eine gewisse Quantität Mangansalz vorhanden sein muls, um einen regelmälsigen Verlauf der Reaktion zu bewirken; ist die vorhandene Salzsäuremenge nur gering - d. h. beträgt sie etwa nur soviel, wie sich bei der Zersetzung von ein oder zwei Gramm Tellurhalogen bildet -- so ist ihr störender Einflufs unter den gewöhnlichen Versuchsbedingungen fast unmerkbar, aber auch in diesem Falle ist es besser, Mangansalz zuzusetzen, weil dann die 'Titration der Oxalsäure bei gewöhnlicher Temperatur ausgeführt werden kann.

1 I. c. S. 241.

Ann. Phys. 118, 48 und 119, 225-226.

${ }^{3}$ Ann. Chem. 213, 302.

4 Amer. Journ. Se. 7, 461. 
In der folgenden Tabelle sind die Resultate einiger Versuche, die mit und ohne Zusatz von Mangansalzen ausgeführt wurden, zusammengestellt.

Tabelle I.

$0=16 . \quad T e=127.5$. Anfangsvolumen $150 \mathrm{ccm}$.

\begin{tabular}{c|c|c|c|c|c}
\hline $\begin{array}{c}\text { Angewandtes } \\
\mathrm{TeO}_{2}\end{array}$ & $\mathrm{NaCl}$ & $\begin{array}{c}\mathrm{H}_{2} \mathrm{SO}_{4} \\
1: 1\end{array}$ & $\begin{array}{c}\mathrm{MnCl}_{2}, \\
4 \mathrm{H}_{2} \mathrm{O}\end{array}$ & $\begin{array}{c}\text { Gefund. } \\
\mathrm{TeO}_{2}\end{array}$ & Fehler \\
$\mathrm{g}$ & $\mathrm{g}$ & $\mathrm{ccm}$ & $\mathrm{g}$ & $\mathrm{g}$ & $\mathrm{g}$ \\
\hline
\end{tabular}

A.

Temperatur bei der Titration $60-80^{\circ} \mathrm{C}$.

\begin{tabular}{l|l|l|l|l|r}
\hline \hline 0.1000 & 0.4 & 5 & - & 0.1003 & +0.0003 \\
0.1000 & 0.4 & 5 & - & 0.1000 & 0.0000 \\
0.1000 & 0.4 & 5 & - & 0.1004 & +0.0004 \\
0.1000 & 1.0 & 5 & - & 0.1003 & +0.0003 \\
0.0650 & 1.0 & 5 & - & 0.0653 & +0.0003
\end{tabular}

$B$.

Temperatur bei der Titration $20-26^{\circ} \mathrm{C}$,

\begin{tabular}{l|l|l|l|l|l}
\hline .0700 & 0.4 & 5.7 & 1.0 & 0.0705 & +0.0005 \\
0.0700 & 0.4 & 5.7 & 1.0 & 0.0698 & -0.0002 \\
0.0700 & 0.4 & 5.7 & 0.5 & 0.0701 & +6.0001 \\
0.1000 & 0.4 & 5.7 & 0.5 & 0.1008 & +0.0008
\end{tabular}

Das Tellurdioxyd, welches durch sorgfältiges Erhitzen des krystallisierten basischen Nitrats ${ }^{1}$ erhalten war, wurde in einer geringen Menge Natriumhydroxyd gelöst, dann wurde die angegebene Menge des Halogensalzes hinzugefügt und in das Reaktionsgemisch bis zur Rotfärbung Permanganatlösung ${ }^{2}$ einfliefsen gelassen; die gebildeten höheren Manganoxyde und das überschüssige Permanganat reduzierte man mit einer titrierten Ammonoxalatlösung, nachdem die Lösung erhitzt und mit ca. $5 \mathrm{ccm}$ mehr Schwefelsäure $(1: 1)$, als zux Neutralisation erforderlich waren, versetzt war. Bei den unter $A$ verzeichneten Versuchen wurde die Flüssigkeit zur Lösung der Oxyde bis auf $60-80^{\circ} \mathrm{C}$. erhitzt und die letzte Titration begann bei der gleichen Temperatur. Bei den Versuchen der Reihe $B$ wurde noch Manganchlorid ( $0.5 \mathrm{~g})$ hinzugefügt, so dals die Reduktion

' Das basische Nitrat wurde durch Oxydation von Tellur mit Salpetersäure gewonnen.

${ }^{2}$ Die Permanganatlösung wurde gegen Ammonoxalat eingestellt. 
der Manganoxyde und die schlielsliche Titration der Oxalsäure bei gewöhnlicher Zimmertemperatur stattfinden konnte.

Die Gegenwart des Chlorids verursacht offenbar bei dieser Methode zur Bestimmung des Tellurs keinerlei Störung, gleichgültig, ob die Titration in der Hitze oder in der Kälte vorgenommen wird.

Bei der weiteren Untersuchung zeigte sich, dals auch nach der gleichen Methode in Gegenwart eines Bromids ziemlich befriedigende Bestimmungen der tellurigen Säure ausgeführt werden können, vorausgesetzt, dafs man die Titration in Gegenwart einer hinreichenden Mangansalzmenge $(0.5-1 \mathrm{~g})$ bei gewöhnlicher Temperatur vornimmt, und dals kein grölserer Schwefelsäureüberschufs als $5 \mathrm{ccm}$ einer $12.5 \%$ igen Mischung vorhanden ist. Bei erhöhter Temperatur wird durch das Permanganat sogleich Brom frei gemacht. Die Resultate der Versuche sind in Tabelle II zusammengestellt.

\section{Tabelle II.}

$0=16 . \quad T e=127.5 . \quad$ Anfangsvolumen $150 \mathrm{~cm}$.

Temperatur bei der Titration $24-26^{\circ} \mathrm{C}$.

\begin{tabular}{|c|c|c|c|c|c|c|}
\hline $\begin{array}{l}\text { Angewandtes } \\
\mathrm{TeO}_{2}\end{array}$ & $\mathrm{NaCl}$ & $\mathrm{KBr}$ & $\begin{array}{c}\mathrm{H}_{2} \mathrm{SO}_{4} \\
12^{1 / 9} / \%\end{array}$ & $\underset{4 \mathrm{H}_{2} \mathrm{O}}{\mathrm{MnCl}_{2}}$ & $\begin{array}{c}\text { Gefundenes } \\
\text { TeO }\end{array}$ & Fehler \\
\hline $\mathrm{g}$ & $g$ & $\mathrm{~g}$ & $\mathrm{ccm}$ & $g$ & g & $g$ \\
\hline$[0.1000$ & $\ldots$ & 0.5 & 20 & 1.0 & 0.1022 & +0.0022 \\
\hline 0.3000 & 一 & 1.5 & 25 & 1.0 & 0.3030 & +0.0030 \\
\hline 0.0650 & - & 0.5 & 1 & 1.0 & 0.0661 & +0.0011 \\
\hline 0.0650 & - & 0.5 & 1 & 1.0 & 0.0647 & -0.0003 \\
\hline 0.1000 & $\ldots$ & 0.5 & 1 & 1.0 & 0.1002 & +0.0002 \\
\hline 03000 & - & 0.5 & 5 & 0.5 & 0.3010 & +0.0010 \\
\hline 0.0650 & 0.5 & 0.5 & 1 & 1.0 & 0.0661 & +0.0011 \\
\hline
\end{tabular}

Aus den angeführten Versuchen geht hervor, dafs man tellurige Säure mit ziemlicher Genauigkeit titrimetrisch durch Kaliumpermanganat in Gegenwart von Chloriden und Bromiden bestimmen kann, wenn man die erste Oxydation in alkalischer Lösung ausführt und die letzte Titration der überschüssigen Oxalsäure bei gewöhnlicher Temperatur in Gegenwart von Mangansalzen mit Zusatz beschränkter Schwefelsäuremengen vornimmt.

In Gegenwart eines Jodids ist die Sachlage anders. Beim Ansäuern des Gemisches des Jodids mit den höheren Oxyden des Mangans, die sich bei der Einwirkung des Permanganats auf die Lösung gebildet haben, wird sofort Jod frei gemacht; dieses Jod 
kann durch Oxalsäure nicht in Jodwasserstoff zurückverwandelt werden. In Gegenwart eines Überschusses von Kaliumjodid werden die sämtlichen höheren Manganoxyde mit grofser Geschwindigkeit reduziert, und das freigemachte Tod ist dann ein Mals für das nach der Oxydation der tellurigen Säure bleibende Permanganat; die Differenz zwischen der ganzen Menge des zugesetzten Permanganats und der so angezeigten nicht verbrauchten Menge giebt dann ein Mals für die tellurige Säure.

Auf dieser Basis haben NorRIs und $F_{A Y}{ }^{1}$ ihre ausgezeichnete jodometrische Bestimmungsmethode für tellurige Säure aufgebaut. Ihr Verfahren besteht darin, dafs die alkalische Lösung der tellurigen Säure mit einer Permanganatlösung von bestimmtem Gehalt solange versetzt wird, bis der Meniskus der Flüssigkeit sich rötlich färbt; dann wird die Lösung mit Eiswasser verdünnt, mit Jodkalium und Schwefelsäure versetzt und mit Thiosulfat titriert. Die Resultate sind ausgezeichnet.

Es ist klar, dafs irgend ein Körper, der das Jod in Jodwasserstoffsäure zurückverwandeln würde, ohne gleichzeitig die Tellursäure wieder zu reduzieren, dazu geeignet wäre, den Überschufs des Permanganats und dadurch die ursprünglich vorhandene tellurige Säure $\mathrm{zu}$ bestimmen.

Wir haben nun gefunden, dafs eine $1 / 10^{-n o r m}$. Arsenitlösung, die, wie üblich, durch Lösen von $4.95 \mathrm{~g}$ reinen, resublimierten Arseniks in einem Liter bikarbonathaltigen Wassers hergestellt war, für unsere Zwecke sehr brauchbar war; diese Lösung besals überdies noch den Vorteil, dafs man sie gleichzeitig auch zur Einstellung des Permanganattiters benutzen kann, und zwar in der Weise, dals man ein abgemessenes Volumen der Permanganatlösung (annäherend $1 / 10^{-n o r m .)}$ in eine mit $2-3 \mathrm{ccm}$ verdünnter Schwefelsäure angesäuerte Kaliumjodidlösung $(1 \mathrm{~g})$ einfliefsen lälst und dann nach der Neutralisation mit Kaliumbikarbonat das freigemachte Jod mit der Lösung der arsenigen Säure bestimmt. Bei dieser Titration mit Jod haben wir die Anwendung von Stärke zur Erkennung der Endreaktion ganz aufgegeben. Die Farbe des freien Jods genügt - sogar bei einer Verdünnung von $300 \mathrm{ccm}$ - zur Erkennung des Endes der Titration und das Verschwinden der gelben Farbe erkennt man bei arseniger Säure ohne Stärke weit schärfer als mit Stärke.

In Tabelle III sind die auf diese Weise erhaltenen Resultate zusammengestellt. $\mathrm{Zu}$ der alkalischen Lösung der tellurigen Säure

${ }^{1}$ Amer. Chem. Journ. 20, 278. 
wurde Jodkalium $(0.5-1 \mathrm{~g}$ auf $100 \mathrm{ccm}$ Wasser) zugesetzt; dann liefs man die titrierte Permanganatlösung einfliefsen, bis die grüne Farbe des Manganats verschwand (ca. $30 \mathrm{ccm}$ der $1 / 10^{-n o r m . ~ L o ̈ s u n g ~}$ für je $0.1 \mathrm{~g} \mathrm{TeO}_{2}$ ); nach dem Zusatz einiger Kubikcentimeter Schwefelsäure klärte sich die Flüssigkeit, wobei sich freies Jod bildete; dieses letztere wurde unter Zusatz von Kaliumbikarbonat titrimetrisch mit arseniger Säure bestimmt. Es ist wichtig, dafs beim Ansäuern der Lösung eine hinreichende Jodkaliummenge vorhanden ist, damit bei der Reduktion der höheren Oxyde des Mangans nicht ein Verlust an Sauerstoff eintritt. $\mathrm{Zu}$ diesem $\mathrm{Zwecke}$ kann man auch die arsenige Säure vor dem Zusatz der Schwefelsäure hinreichend zugeben. Dies letztere Verfahren ist dann von Vorteil, wenn man aus irgend einem Grunde nicht mehr Jod in das Reaktionsgemisch hineinbringen will als ursprünglich darin vorhanden war, z. B. wenn nach der Bestimmung der tellurigen Säure noch eine Bestimmung des Jods folgen soll.

Tabelle III.

$\mathrm{O}=16 . \quad \mathrm{Te}=127.5$.

\begin{tabular}{|c|c|c|c|c|c|c|c|}
\hline $\begin{array}{c}\text { Angew. } \\
\mathrm{TeO}_{2} \\
\mathrm{~g} \\
\end{array}$ & $\begin{array}{c}\mathrm{NaCl} \\
\mathrm{g} \\
\end{array}$ & $\begin{array}{c}\mathrm{KBr} \\
\mathrm{g}\end{array}$ & $\begin{array}{c}\mathbf{K J} \\
\mathrm{g} \\
\end{array}$ & $\begin{array}{c}\text { Gesamtes } \\
\text { Endvol. } \\
\text { ccm }\end{array}$ & $\begin{array}{l}\text { Bei d. Oxyda } \\
\text { tion vorhand. } \\
\mathrm{NaOH} \\
\mathrm{g}\end{array}$ & $\begin{array}{c}\text { Gefunden. } \\
\mathrm{FeO}_{2} \\
\mathrm{~g}\end{array}$ & $\begin{array}{c}\text { Fehler } \\
\mathbf{g}\end{array}$ \\
\hline 0.1000 & - & - & 0.5 & 160 & 0.1 & 0.1005 & +0.0005 \\
\hline 0.1000 & - & 一 & 0.5 & 160 & 0.1 & 0.1001 & +0.0001 \\
\hline 0.1000 & - & - & 0.5 & 160 & 0.1 & 0.1003 & +0.0003 \\
\hline 0.1000 & - & - & 1.0 & 250 & 0.1 & 0.1007 & +0.0007 \\
\hline 0.2000 & - & - & 1.0 & 250 & 0.2 & 0.1997 & -0.0003 \\
\hline 0.1000 & 0.5 & 0.5 & 0.5 & 250 & 0.1 & 0.1000 & 0.0000 \\
\hline 0.2100 & 1.0 & 1.0 & 1.0 & 225 & 0.2 & 0.2105 & +0.0005 \\
\hline 0.1000 & - & - & 0.5 & 160 & 1.0 & 0.1011 & +00011 \\
\hline 0.2000 & - & - & 1.0 & 300 & 2.0 & 0.2009 & +0.0009 \\
\hline
\end{tabular}

Diese Resultate sind ziemlich befriedigend. Ebenso wie die Werte von Tabelle I würden sie sich praktisch auf den von der Theorie verlangten Durchschnitt bringen lassen, wenn an Stelle des von Clarke und Richards angegebenen Wertes $\mathrm{Te}=127.5$ der von dem Komitee der Deutschen Chemischen Gesellschaft vorgeschlagene Wert $\mathrm{Te}=127$ angewendet würde.

The Kent Chemical Laboratory of Yale University, New Haven, U. S. A.

Bei der Redaktion eingegangen am 8. Juli 1899. 